\title{
Remdesivir, Molnupiravir and Nirmatrelvir remain active against SARS-CoV-2 Omicron and other variants of concern
}

\author{
Laura Vangeel $^{\mathrm{a}}$, Winston Chiu ${ }^{\mathrm{a}}$, Steven De Jonghe ${ }^{\mathrm{a}}$, Piet Maes ${ }^{\mathrm{b}}$, Bram Slechten ${ }^{\mathrm{c}}$, \\ Joren Raymenants $^{c, d}$, Emmanuel André ${ }^{c, d}$, Pieter Leyssen ${ }^{a}$, Johan Neyts ${ }^{\text {a, }, \text {, , }}$, \\ Dirk Jochmans ${ }^{\text {a, } 1 \text {,* }}$ \\ ${ }^{\text {a }}$ KU Leuven, Department of Microbiology, Immunology and Transplantation, Rega Institute, Laboratory of Virology and Chemotherapy, Leuven, Belgium \\ ${ }^{\mathrm{b}}$ KU Leuven, Department of Microbiology, Immunology and Transplantation, Rega Institute, Laboratory of Clinical and Epidemiological Virology, Leuven, Belgium \\ ${ }^{\mathrm{c}}$ University Hospitals Leuven, Department of Laboratory Medicine, Leuven, Belgium \\ ${ }^{\mathrm{d}}$ KU Leuven, Department of Microbiology, Immunology and Transplantation, Rega Institute, Laboratory of Clinical Bacteriology and Mycology, Leuven, Belgium
}

\section{A B S T R A C T}

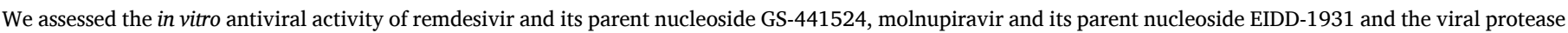

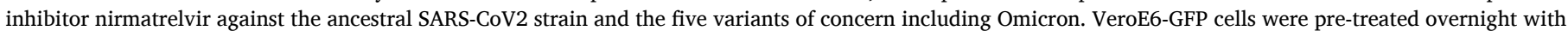

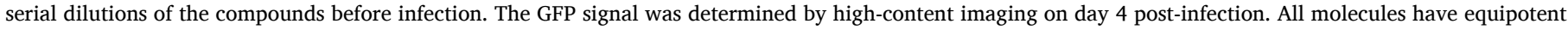

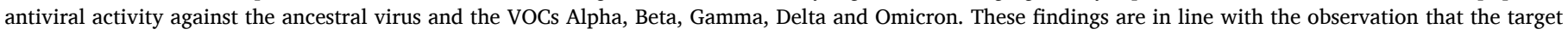
proteins of these antivirals (respectively the viral RNA dependent RNA polymerase and the viral main protease Mpro) are highly conserved.

One and a half year after the start of the global COVID-19 pandemic caused by severe acute respiratory syndrome coronavirus 2 (SARS-CoV2), multiple variants have emerged. These can be harmless or slightly beneficial for the virus, causing for example increased transmission, virulence, or immune escape (Davies et al., 2021; Sabino et al., 2021; Mahase, 2021). SARS-CoV-2 genetic diversification was initially considered slow when the virus was spreading in early 2020 . The first official variant, a single spike D614G mutation found in early European lineages, was linked to more efficient transmission (Volz et al., 2021) and rapidly spread to become the dominant viral strain worldwide. Late 2020, multiple variants emerged that spiked regional epidemics. Five 'variants of concern' (VOC) have been identified (Alpha, Beta, Gamma, Delta and Omicron). All have characteristic mutations (www.ecdc.eu ropa.eu/en/covid-19/variants-concern). The spike (S) glycoprotein appears especially prone to accumulate mutations (Saputri et al., 2020) and all of the circulating VOCs have some mutations that favor evasion from the host immune response (Khateeb et al., 2021). Numerous spike-protein based vaccines were developed and vaccination programs are running at full speed. However, studies of sera and emerging real-world evidence indicate that Omicron escapes the immunity whether from previous infection or vaccination (Cohen, 2021).
Several direct-acting antivirals against SARS-CoV-2 have been approved or are advancing in clinical development. They can be divided in two classes, monoclonal antibodies (mAbs) directed against the Spike protein and small molecules interfering with the viral replication machinery. mAbs are administered intravenously but studies are underway to explore intramuscular or subcutaneous administration which would overcome the requirement of a hospital setting for dosing. (Kumar et al., 2021). Recent cell culture data indicates that the SARS-CoV-2 variant of concern (VOC) Omicron is not susceptible to most of the approved mAbs making it unlikely that their clinical efficacy will be maintained (Alexander Wilhelm et al., 2021; VanBlargan et al., 2022).

The direct-acting small-molecule SARS-CoV-2 antivirals that have received approval or emergency use authorization do not target the variable spike-protein but target either the conserved viral RNAdependent RNA polymerase (RdRp) or the conserved viral main protease (Mpro or 3CL protease). Remdesivir, a monophosphoramidate prodrug of the nucleoside GS-441524, originally developed to treat Ebola virus infections, inhibits the RdRp of SARS-CoV-2. It was the first antiviral approved or authorized for emergency use to treat COVID-19 in several countries. Remdesivir improves clinical outcomes in patients hospitalized with moderate-to-severe disease and it prevents disease

\footnotetext{
* Corresponding author.

** Corresponding author.

E-mail addresses: johan.neyts@kuleuven.be (J. Neyts), dirk.jochmans@kuleuven.be (D. Jochmans).

1 These authors have contributed equally to this work and share last authorship.
} 


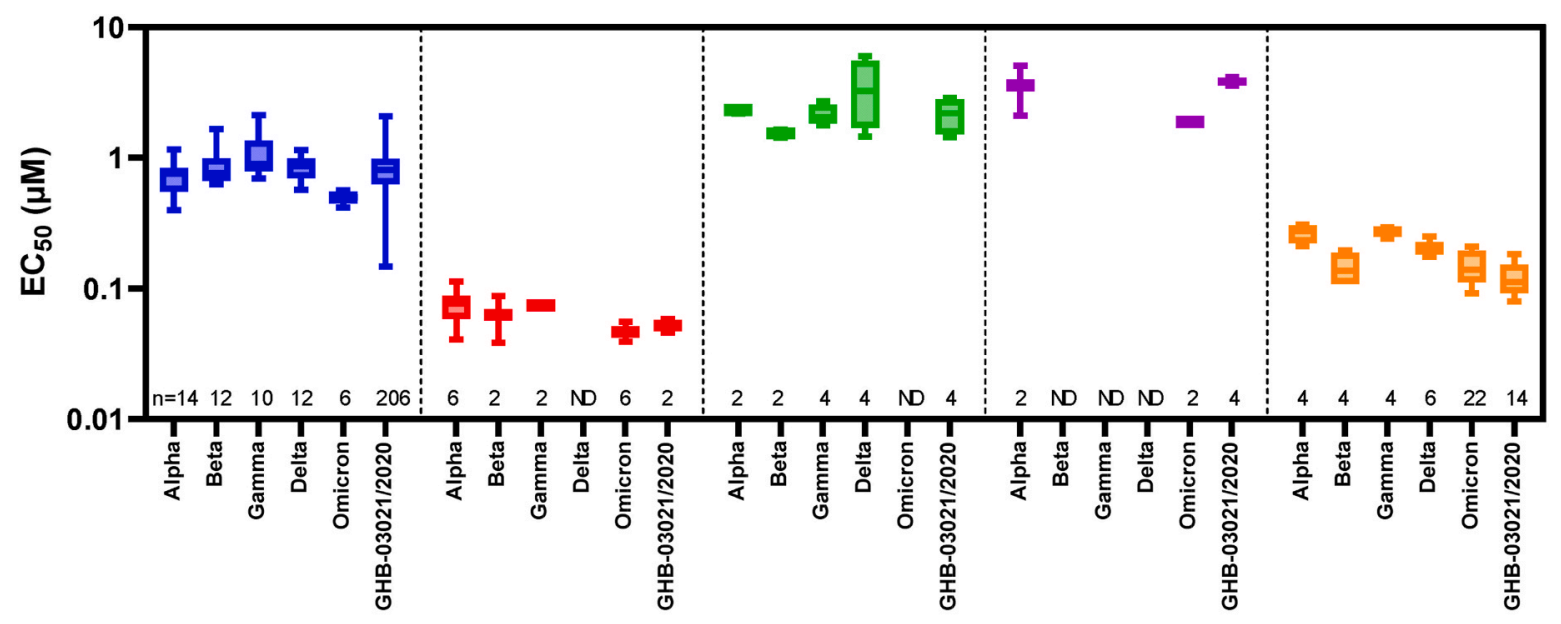

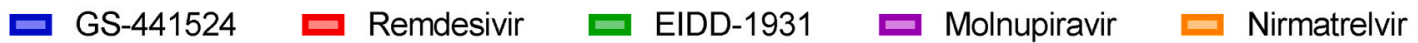

Fig. 1. Activity of various antivirals upon infection of VeroE6-GFP cells with different SARS-CoV-2 VOC. VeroE6-GFP cells were pre-treated overnight with serial dilutions of the compounds. The next day, cells were infected with SARS-CoV-2 at a multiplicity of infection (MOI) of 0.001 tissue culture infectious dose (TCID 50 ) per cell. The number of fluorescent pixels of GFP signal, determined by high-content imaging on day 4 post-infection, was used as read-out. The percentage of inhibition was calculated by subtracting background (number of fluorescent pixels in the untreated infected control wells) and normalizing to the untreated-uninfected control wells (also background subtracted). The $50 \%$ effective concentration $\left(\mathrm{EC}_{50}\right.$, the concentration of compound required for fifty percent recovery of cell-induced fluorescence) was determined using logarithmic interpolation. These experiments were performed in the presence of the Pgp-inhibitor CP-100356 (0.5 $\mu \mathrm{M})$ in order to limit compound efflux. This graph was created using Graphpad Prism 9.2.0. The boxes extend from the 25th to 75th percentiles while the whiskers indicate the minimal and maximal values. The numbers above the $\mathrm{X}$-axis indicate the number of measurements for each condition. While we determined the $\mathrm{EC}_{50}$ of remdesivir, molnupiravir and nirmatrelvir on Omicron we did not determine the $\mathrm{EC}_{50}$ on all VOC for all compounds tested. Due to time constrains we only used historical data from our database for the other VOCs and thus some of the values are depicted as "ND" ("Not Determined"). For the same reason, we included EIDD1931 to allow comparison with molnupiravir as both compounds are intracellularly converted to the same antiviral molecule and thus have the same $\mathrm{EC}_{50}$. The individual $\mathrm{EC}_{50} \mathrm{~s}$ values of this study are available at Mendeley Data (https://doi.org/10.17632/bmjc74dyjs.1).

progression in outpatients (Beigel et al., 2020; Gottlieb et al., 2021). While remdesivir requires intravenous administration, an oral prodrug of GS-441524 is being developed (Cox et al., 2021). Molnupiravir (MK-4482 or EIDD-2801), a prodrug of the nucleoside analogue EIDD-1931 ( $\beta$-D-N4-hydroxycytidine), is another inhibitor of the viral RdRp and was originally developed against different RNA viruses such as influenza (Painter et al., 2021). A phase 2a clinical trial of molnupiravir in patients with COVID-19 shows accelerated SARS-CoV-2 RNA clearance and elimination of infectious virus (Fischer et al., 2021). This orally bioavailable drug was recently authorized in the UK for use in people who have mild to moderate COVID-19 and who have at least one risk factor for developing severe illness. Also, the U.S. FDA issued an emergency use authorization (EUA) in infected adults who are at high risk for progression to severe COVID-19, and for whom alternative COVID-19 treatment options are not accessible or clinically appropriate.

Another target for antiviral drugs is the viral main protease Mpro (or 3CL protease), a cysteine protease which cleaves the two polyproteins (pp1a and pp1ab) of SARS-CoV-2 at multiple locations, resulting in the various non-structural proteins, which are key for viral replication. Nirmatrelvir (PF-07321332), is an irreversible inhibitor of SARS-CoV-2 Mpro that is co-formulated with ritonavir allowing an oral route of administration (known as Paxlovid). When treatment is initiated during the first days after symptom onset, it results in roughly a $90 \%$ protection against severe COVID-19 and hospitalization (Owen et al., 2021). Even though the Mpro-gene can be slightly affected by evolutionary mutations, the antiviral potency does not seem to be compromised (Sven Ullrich et al., 2021).

We here assess the in vitro antiviral effect of GS-441524, remdesivir, EIDD-1931, molnupiravir and nirmatrelvir against the various SARSCoV-2 VOCs, including Omicron.

The SARS-CoV-2 antiviral assay is based on the previously established SARS-CoV assay(Ivens et al., 2005). Upon infection the fluorescence of VeroE6-GFP cell cultures declines due to a cytopathogenic effect. In the presence of an antiviral compound, the cytopathogenicity is inhibited and the fluorescent signal maintained. To this end VeroE6-GFP cells (kindly provided by Marnix Van Loock, Janssen Pharmaceutica, Beerse, Belgium), were used as described previously (Do et al., 2021; Rana Abdelnabi et al., 2021). Since VeroE6 cells show a high efflux of some chemotypes, the antiviral assays were performed in the presence of the P-glycoprotein (Pgp) efflux inhibitor CP-100356 (0.5 $\mu \mathrm{M})($ Hoffman et al., 2020). A SARS-CoV-2 strain grown from the first Belgian patient sample (GHB-03021/2020), was used as ancestral strain as it is closely related to the prototypic Wuhan-Hu-1 2019-nCoV (GenBank accession number MN908947.3) (Boudewijns et al., 2020). All the other isolates were obtained from patients in Belgium and more information can be found in GISAID (Alpha = EPI_ISL_791333; Beta = EPI_ISL_896474; Gamma = EPI_ISL_1091366; Delta = EPI_ISL_2425097; Omicron = EPI_ISL_6794907). The multiplicity of infection (MOI) was kept constant for the different VOC to allow comparison of the potency.

Our in vitro results show that GS-441524, remdesivir, EIDD-1931, molnupiravir and nirmatrelvir retain their activity against all current VOCs including Omicron (Fig. 1). The maximal change of the median $\mathrm{EC}_{50} \mathrm{~S}$ over the different VOC is for each compound $<3 \mathrm{x}$ (1.8x for GS441524, 1.6x for remdesivir, 2.5x for the series EIDD-1931 and molnupiravir and $2.5 \mathrm{x}$ for nirmatrelvir). In our experience these results are within the normal range of measurement error. For example, the ratio between the $95 \%$ and $5 \%$ percentile of the $\mathrm{EC}_{50}$ s of GS-441524 on GHB$03021 / 2020$ is $2.9 x$ ( $n=206$; calculated using Graphpad v9.2.0). So only differences in $\mathrm{EC}_{50} \mathrm{~S}$ of $>3 \mathrm{x}$ and with statistical significance should be considered as meaningful differences using this methodology. The individual EC50s values of this study are available at Mendeley Data (https://doi.org/10.17632/bmjc74dyjs.1).

The fact that these antivirals retain their activity on the different SARS-CoV-2 VOCs is in accordance with the observation that the target proteins of these antivirals are highly conserved. For the RdRp there are only two amino acid changes (P323L in all VOCs and G671S in Delta; 
position $4715 / 5063$ in ORF1ab or $314 / 662$ in ORF1b respectively) when compared with the ancestral lineage (NC_045512). As these are distant from the active site, a different susceptibility towards remdesivir or molnupiravir is not to be expected. For the Mpro there are also two amino acid changes described (K90R in Beta and P132H in Omicron; position 3353 and 3395 in ORF1ab respectively). Alike for the RdRp, these mutations are not located near the active site of the Mpro and hence no difference in susceptibility for nirmatrelvir is expected.

These results indicate that when more VOCs arise, due to antigenic drift, there is a high probability that they will remain sensitive towards current (and likely also future) antivirals that do not target the spike. It is therefore of utmost importance to develop more pan-corona antivirals as they will be an essential armor and complement vaccines in the strategy to control the current pandemic (Torneri et al., 2020).

\section{Declaration of interests}

The authors declare that they have no known competing financial interests or personal relationships that could have appeared to influence the work reported in this paper.

\section{Acknowledgements}

We thank Lize Cuypers, Guy Baele, Simon Dellicour and the Belgian Genomic Surveillance program which contributed to the early detection of Omicron in Belgium. We thank Tina Van Buyten, Birgit Voeten, Joost Schepers, Thibault Francken, Kim Donckers, and Niels Cremers for excellent technical assistance. We thank Fran Berlioz-Seux, Betsy Russel and Rob Jordan for helpful discussion. Part of this research work was performed using the 'Caps-It' research infrastructure (project ZW13-02) that was financially supported by The Hercules Foundation, Belgium and Rega Foundation, KU Leuven. This project has received funding from the European Health Emergency preparedness and Response Authority (HERA), the Covid-19-Fund KU Leuven/UZ Leuven and the COVID-19 call of FWO, Belgium (G0G4820N), the European Union's Horizon 2020 research and innovation program under grant agreements No 101003627 (SCORE project) and Bill \& Melinda Gates Foundation (BGMF) under grant agreement INV-00636 and the Innovative Medicines Initiative 2 Joint Undertaking (JU) under grant agreement No 101005077. The JU receives support from the European Union's Horizon 2020 research and innovation program, EFPIA, European Union, the Bill \& Melinda Gates Foundation, the Global Health Drug Discovery Institute, China, and the University of Dundee.

\section{References}

Alexander Wilhelm, M.W., Grikscheit, Katharina, Toptan, Tuna, Schenk, Barbara, Pallas, Christiane, Metzler, Melinda, Kohmer, Niko, Hoehl, Sebastian,
Helfritz, Fabian A., Wolf, Timo, Goetsch, Udo, Ciesek, Sandra, 2021. Reduced neutralization of SARS-CoV-2 omicron variant by vaccine sera and monoclonalantibodies. medRxiv. https://doi.org/10.1101/2021.12.07.21267432.

Beigel, J.H., et al., 2020. Remdesivir for the treatment of covid-19 - final report. N. Engl. J. Med. 383 (19), 1813-1826.

Boudewijns, R., et al., 2020. STAT2 signaling restricts viral dissemination but drives severe pneumonia in SARS-CoV-2 infected hamsters. Nat. Commun. 11 (1), 5838.

Cohen, J., 2021. Omicron sparks a vaccine strategy debate. Science 374 (6575), 1544-1545.

Cox, R.M., et al., 2021. Oral prodrug of remdesivir parent GS-441524 is efficacious against SARS-CoV-2 in ferrets. Nat. Commun. 12 (1), 6415.

Davies, N.G., et al., 2021. Estimated transmissibility and impact of SARS-CoV-2 lineage B.1.1.7 in England. Science (6538), 372.

Do, T.N.D., et al., 2021. A robust SARS-CoV-2 replication model in primary human epithelial cells at the air liquid interface to assess antiviral agents. Antivir. Res. 192, 105122.

Fischer 2nd, W.A., et al., 2021. A Phase 2a clinical trial of Molnupiravir in patients with COVID-19 shows accelerated SARS-CoV-2 RNA clearance and elimination of infectious virus. Sci. Transl. Med. eabl7430.

Gottlieb, R.L., et al., 2021. Early remdesivir to prevent progression to severe covid-19 in outpatients. N. Engl. J. Med.

Hoffman, R.L., et al., 2020. Discovery of ketone-based covalent inhibitors of coronavirus 3CL proteases for the potential therapeutic treatment of COVID-19. J. Med. Chem. 63 (21), 12725-12747.

Ivens, T., et al., 2005. Development of a homogeneous screening assay for automated detection of antiviral agents active against severe acute respiratory syndromeassociated coronavirus. J. Virol. Methods 129 (1), 56-63.

Khateeb, J., Li, Y., Zhang, H., 2021. Emerging SARS-CoV-2 variants of concern and potential intervention approaches. Crit. Care 25 (1), 244.

Kumar, S., Chandele, A., Sharma, A., 2021. Current status of therapeutic monoclonal antibodies against SARS-CoV-2. PLoS Pathog. 17 (9), e1009885.

Mahase, E., 2021. Covid-19: Novavax vaccine efficacy is $86 \%$ against UK variant and $60 \%$ against South African variant. BMJ 372, n296.

Owen, D.R., et al., 2021. An oral SARS-CoV-2 M(pro) inhibitor clinical candidate for the treatment of COVID-19. Science eabl4784.

Painter, W.P., et al., 2021. Human safety, tolerability, and pharmacokinetics of molnupiravir, a novel broad-spectrum oral antiviral agent with activity against SARS-CoV-2. Antimicrob. Agents Chemother.

Rana Abdelnabi, C.S.F., Jochmans, Dirk, Vangeel, Laura, De Jonghe, Steven, Augustijns, Patrick, Mols, Raf, Weynand, Birgit, Wattanakul, Thanaporn, Hoglund, Richard M., Tarning, Joel, Mowbray, Charles E., Peter, Sjö, Escudié, Fanny, Ivan Scandale, Chatelain, Eric, Neyts, Johan, 2021. The oral protease inhibitor (PF07321332) protects Syrian hamsters against infection with SARS-CoV-2 variants of concern. bioRxiv.

Sabino, E.C., et al., 2021. Resurgence of COVID-19 in Manaus, Brazil, despite high seroprevalence. Lancet 397, 452-455, 10273.

Saputri, D.S., et al., 2020. Flexible, functional, and familiar: characteristics of SARS-CoV2 spike protein evolution. Front. Microbiol. 11, 2112.

Sven Ullrich, K.B.E., Gottfried, Otting, Nitsche, Christoph, 2021. Main protease mutants of SARS-CoV-2 variants remain susceptible to PF-07321332. bioRxiv.

Torneri, A., et al., 2020. A prospect on the use of antiviral drugs to control local outbreaks of COVID-19. BMC Med. 18 (1), 191.

Volz, E., et al., 2021. Evaluating the effects of SARS-CoV-2 spike mutation D614G on transmissibility and pathogenicity. Cell 184 (1), 64-75 e11.

VanBlargan, L. A., Errico, J. M., Halfmann, P. J., Zost, S. J., Crowe, J. E., Jr, Purcell, L. A., Kawaoka, Y., Corti, D., Fremont, D. H., \& Diamond, M. S. (2022). An infectious SARS-CoV-2 B.1.1.529 Omicron virus escapes neutralization by therapeutic monoclonal antibodies. Nature medicine, 1-6. Advance online publication. https:// doi.org/10.1038/s41591-021-01678-y. 